\section{Prácticas de sostenibilidad ambiental y energética: experiencias con una escuela agraria en el noroeste de la provincia de Buenos Aires (Argentina)}

\section{Melina Yuln}

Universidad Nacional del Noroeste

de la Provincia de Buenos Aires, Argentina.

myuln@comunidad.unnoba.edu.ar

(iD) orcid.org/0000-0003-3177-7034
Ambiente y extensión universitaria /

Intervenciones
RECEPCIÓN: 12/03/21

ACEPTACIÓN FINAL: 17/05/21
Energy sustainability practices: experiences with an agrarian school in the Northwest of Buenos Aires province (Argentina)

\section{Abstract}

This article presents the experience of a university extension project aimed at implementing sustainability practices in a Secondary School of agrarian training. The role of university extension and the environmental issue is put into context from the National University of the Northwest of the Province of Buenos Aires (UNNOBA), as well as the positioning of the extension team, coming from the field of research. The proposal and the impact of the project will be developed both within the university team and its link with the educational community. Some conclusions will be tested, highlighting the incorporation of sustainable practices and actions, in educational settings, to collaborate with objectives to combat climate change, increase energy security and promote social inclusion.

Keywords: sustainability; education; environment; energy; university extension.
Práticas de sustentabilidade energética: experiências com uma escola agrícola no noroeste da província de Buenos Aires (Argentina)

\section{Resumo}

Este artigo apresenta a experiência de um projeto de extensão universitária que visa implementar práticas de sustentabilidade em uma escola de ensino médio de formação agrícola. Para isso, o papel da extensão universitária e a questão ambiental são colocados no contexto da Universidade Nacional do Noroeste da província de Buenos Aires (UNNOBA), bem como o posicionamento da equipe de extensão, vindo do campo de pesquisa. A proposta e o impacto do projeto serão desenvolvidos tanto no seio da equipe universitária quanto no seu vínculo com a comunidade educacional. Algumas conclusões serão testadas, destacando-se a incorporação de práticas e ações sustentáveis, em ambientes educacionais, para colaborar com os objetivos de combate às mudanças climáticas, aumento da segurança energética e promoção da inclusão social.

Palavras-chave: sustentabilidade; educação; meio ambiente; energia; extensão universitária.

Palabras clave: sostenibilidad; educación ambiente; energía; extensión universitaria. 


\section{Introducción}

Desde 2015, los Objetivos de Desarrollo Sostenible (ODS) han ingresado en las agendas de gobiernos, instituciones y empresas. A partir de un conjunto de objetivos globales acordados entre diversos países y con el respaldo de Naciones Unidas, se establecieron metas ambientales, sociales y económicas por alcanzar hasta 2030. Esas metas se insertan en objetivos específicos que buscan erradicar la pobreza, proteger al planeta y asegurar la paz y prosperidad de la población mundial. Buena parte de lo ODS se orienta al cuidado ambiental, en términos amplios, y avanza en el camino trazado por estrategias internacionales para la mitigación del cambio climático.

Siguiendo objetivos globales de sostenibilidad ambiental, se valorizan acciones a escala local que motivan iniciativas múltiples. La sostenibilidad es entendida como la capacidad de satisfacer las necesidades de las generaciones presentes sin transferir perjuicios en el espacio geográfico, ni en el tiempo, ni entre individuos o grupos sociales (Organización de la Naciones Unidas [ONU], 1987; Kozak y Romanello, 2012). Estrategias de sostenibilidad, como el uso racional y eficiente de los recursos y la incorporación de fuentes renovables de energía, se proponen para reducir las emisiones de gases efecto invernadero y la consecuente dependencia mundial respecto de los hidrocarburos.

Las acciones de eficiencia energética mejoran las condiciones del hábitat y la disponibilidad de recursos, el bienestar social y económico (Thema et al., 2017). La eficiencia energética promueve a su vez la equidad social puesto que contribuye a que las poblaciones menos favorecidas tengan acceso a servicios e infraestructuras seguros y de calidad (Carrizo y Gil, 2018). La incorporación de fuentes renovables de energía se presenta como otra alternativa de sostenibilidad para el aprovechamiento de recursos locales: solares, eólicos, hidráulicos, biomasa y geotérmicos. Asimismo, contribuyen a la multiplicación de empleos.

En Argentina, las energías renovables no convencionales aportan valores en torno al $10 \%$ de la energía primaria consumida; mientras que el recurso gasífero aporta más de la mitad de la energía que requiere el país y el petróleo más de un tercio. En el siglo XXI aparecen nuevos aportes a la matriz energética a través del corte de combustibles fósiles con biocombustibles, de los parques eólicos de alta potencia y de la energía solar, la biomasa y el biogás.

Gran parte de la población rural se ve restringida en cuanto a utilizar formas de energía tradicionales como la leña, gas envasado, baterías o grupos electrógenos, con costos económicos que las vuelven menos competitivas y con mayores impactos ambientales. No obstante, para que se produzca una transición energética a un sistema sostenible no solo son necesarias políticas públicas efectivas sino también compromiso ciudadano, con cambios de hábitos en el uso responsable y eficiente de la energía. En este escenario, la generación de energía a través del aprovechamiento de recursos situados abre nuevas posibilidades para un sistema energético más inclusivo y sostenible. La asociación y la cooperación de actores locales, como municipios, instituciones y organizaciones, propician estas acciones y prácticas en consonancia con los ODS.

En tanto, ante el desafío que suponen estas iniciativas locales con perspectivas globales, se planteó - como objetivo para el desarrollo de un proyecto de extensión en la Universidad Nacional del Noroeste de la Provincia de Buenos Aires (UNNOBA) - ${ }^{1}$ favorecer prácticas de 
sostenibilidad ambiental y energética en el ámbito escolar, para lo cual se trabajó con una Escuela de Educación Secundaria Agraria en Lincoln, provincia de Buenos Aires, ${ }^{2}$ como institución copartícipe.

En este artículo se expondrá la experiencia de un proyecto de extensión orientado a implementar prácticas de sostenibilidad ambiental y energética. Para ello, el texto se organiza en dos apartados: en el primero se abordará el rol de la extensión universitaria y la preocupación por la cuestión ambiental en UNNOBA, así como el posicionamiento del equipo extensionista, proveniente del ámbito de la investigación. En el segundo apartado, se desarrollará la experiencia con la Escuela Agraria y el impacto del proyecto al interior del espacio extensionista y con la comunidad educativa. Finalmente, se ensayarán algunas conclusiones y se plantearán propuestas de continuidad.

\section{El ámbito institucional de UNNOBA}

La UNNOBA busca estimular y fomentar las actividades y acciones de extensión universitaria con el objeto de contribuir al cumplimiento de su función social así como a su consolidación y jerarquización.

"La Universidad, como un medio de desarrollo social al servicio de la comunidad, contribuye al bienestar de la población, al desarrollo económico sustentable, a la preservación del medio ambiente y al fortalecimiento de la identidad cultural. La función de Extensión tiene como objetivo la interacción con la sociedad para el análisis de las necesidades y problemáticas de la comunidad, promoviendo el desarrollo social, cultural y artístico; para lo cual desarrollará tareas que considere pertinentes incentivando la participación de docentes, estudiantes y graduados. A su vez, la Universidad colaborará a nivel municipal, regional, provincial y nacional en el estudio y aporte a la solución de problemas que afectan a la población. Para ello, se vinculará con la comunidad, las instituciones, y los gobiernos, favoreciendo el intercambio y la transferencia de los conocimientos". (UNNOBA, 2009, p.10)

La extensión universitaria es la actividad mediante la cual la Universidad aporta a la sociedad los resultados y logros de su investigación y docencia. Asimismo, al conocer la realidad social y su cultura, enriquece toda su actividad académica conjunta. Sus funciones son:

- Entender en la elaboración de los proyectos de reglamentaciones y resoluciones del área.

- Entender en la difusión cultural, científica y tecnológica de todo el conocimiento que la Universidad posee, contando para ello con la participación de docentes, graduados y estudiantes.

- Ocuparse de la incorporación al acervo cultural de la Universidad, de los conocimientos y saberes que la sociedad produce, es decir, de su cultura.

- Entender en los requerimientos provenientes de los claustros universitarios en tareas culturales, deportivas o comunitarias.

- Entender en todo lo referente a la prevención y asistencia médica de los claustros universitarios. 
- Asesorar, coordinar y supervisar la tramitación y gestión de todo lo relacionado con las funciones antes mencionadas.

- Promocionar la integración de los estudiantes al medio social universitario, desarrollando en ellos el sentido de solidaridad, responsabilidad y cooperación.

- Promocionar la firma de convenios de cooperación con fundaciones y municipalidades de la región para atender distintas necesidades de los estudiantes.

- Entender en el desarrollo de trabajos de investigación para indagar las problemáticas, necesidades y expectativas de la población en general.

- Promocionar actividades de turismo cultural, social y de recreación destinadas al estudiante en particular y a la comunidad universitaria en general.

- Estimular en los estudiantes una conciencia a favor de la práctica deportiva.

- Asistir a las comisiones que creare el Consejo Superior en el ámbito de su competencia (UNNOBA, s.f.)

Las convocatorias anuales para la presentación de propuestas de proyectos de extensión tienen la finalidad de promover la vinculación de la UNNOBA con la comunidad, entendiendo que un trabajo articulado con sus actores favorecerá el desarrollo y la mejora en la calidad de vida. Desde hace varios años, la instrumentación de estas convocatorias otorga un fuerte impulso a las actividades de extensión universitaria, con el reconocimiento académico y presupuestario de la Universidad. Se privilegia la inserción de estudiantes, docentes, personal no docente y jóvenes graduados en políticas de fortalecimiento de las relaciones de educación superior con el medio, con el sistema socioeconómico, cultural y productivo de la región, y con la realidad cultural de la sociedad.

En este sentido, la extensión se plantea en UNNOBA bajo las premisas del compromiso social universitario, siguiendo los postulados de la Cumbre Mundial de París (Organización de las Naciones Unidas para la Educación, la Ciencia y la Cultura [UNESCO], 1998) respecto de la educación superior y el rol de las universidades en el siglo XXI. La misión social de la universidad se alinea prioritariamente hacia las demandas de la comunidad, atendiendo la consolidación de los derechos humanos, el desarrollo sostenible, la democracia y la paz, en un marco de justicia. De este modo, la propuesta se integra a los ODS con la proposición de ejes temáticos que se enmarcan en cada uno de esos objetivos (ONU, s.f.).

\section{Abordaje institucional de la cuestión ambiental}

La preocupación por la cuestión ambiental en UNNOBA se manifiesta tanto desde lo académico como desde la gestión y la vinculación con la comunidad. La Universidad cuenta con diversas áreas, departamentos y programas orientados a su tratamiento.

Desde el área de "Seguridad, higiene y protección ambiental" se desarrolla un sistema de gestión ambiental que alcanza actividades y servicios educativos universitarios de pregrado, grado y posgrado, extensión, procesos de investigación y vinculación tecnológica, dependencias académicas o administrativas, establecidos en los edificios Eva Duarte de Perón y la biblioteca Silvina Ocampo, de Junín. Para ello se plantean políticas y objetivos ambientales anuales tendientes a crear conciencia acerca de la sustentabilidad ambiental con el objetivo central de incorporarla a los hábitos y conductas de la comunidad universitaria; contribuir a la formación profesional a través de principios y hábitos que permitan al graduado incluir la 
dimensión ambiental en el desarrollo de su vida profesional; utilizar la gestión interna y promover la educación y capacitación para la mejora continua del desempeño ambiental. Asimismo, se busca optimizar el empleo de los recursos naturales; minimizar la cantidad de residuos generados por nuestras actividades, reciclándolos en la medida en que sea posible; prevenir la contaminación y reducir los impactos ambientales. Se establecen anualmente objetivos y metas ambientales y se evalúa su grado de cumplimiento. Entre estos indicadores se destaca el cálculo anual de la huella de carbono, es decir, la incidencia de la actividad universitaria en la generación de emisiones de gases efecto invernadero.

La Universidad también cuenta con un Campo Experimental, un predio de 84 hectáreas con aulas, laboratorios y módulos de producción animal y vegetal donde se desarrollan actividades productivas y de investigación vinculadas a cultivos, energías renovables y producción animal. Allí funcionan la Granja Educativa, el Invernadero y el Laboratorio de Investigación y Desarrollo en Energías Renovables (LIDER). La Granja Educativa consiste en una propuesta para que los estudiantes de distintos niveles y modalidades educativas puedan visualizar y experimentar actividades de huerta, granja y energías renovables. El Invernadero tiene como objetivos transferir tecnologías a los productores hortícolas, generar un volumen de producción de hortalizas que permita industrializarlas a través del "Programa de Alimentos UNNOBA", consolidar proyectos de investigación y actividades académicas del área, así como formar parte del recorrido de la Granja Educativa. LIDER es el laboratorio desde el cual se busca brindar soluciones para los crecientes problemas ambientales dentro de sus distintas áreas energéticas (solar fotovoltaica y térmica, eólica, biomasa, eficiencia energética).

A estas iniciativas de gestión e investigación se suma la incorporación de nuevos contenidos y/o carreras a la oferta académica de grado y posgrado así como la promoción y desarrollo de líneas de investigación en diversas temáticas ambientales.

\section{De la investigación a la extensión}

Los integrantes del equipo extensionista son docentes-investigadores que trabajan de manera colectiva desde UNNOBA y otras universidades e instituciones. ${ }^{3}$ Se conforman equipos multidisciplinarios que combinan capacidades en arquitectura, ingeniería, geografía, docencia, gestión ambiental, hábitat y urbanismo sustentable, etc. Cuentan con experiencia en proyectos de investigación financiados por UNNOBA u otras instituciones respecto de temáticas como territorios, energía y ambiente. En ese marco se aborda la complejidad de los temas, se trabaja sobre dimensiones económicas, sociales, políticas y ambientales, considerando que la apropiación de las tecnologías y la solución a los problemas de la población requieren conocimiento global y tratamiento integral. Con la finalidad de generar conocimiento sobre los temas estudiados para favorecer el desarrollo sostenible, la labor se centra en la comunión de distintas disciplinas y en la interacción de las ciencias con la práctica.

3) Proyectos de Investigación desarrollados en UNNOBA: "Transiciones energéticas y trayectorias territoriales en la Argentina del siglo XXI" (2017 -2018); "En la transición hacia la inclusión y sostenibilidad energética. Experiencias, estrategias y desafíos para Argentina" (2019-202); "Desafíos de Patrimonio y Sostenibilidad en el noroeste de la provincia de Buenos Aires" (2019-2021). 
Y además de generar conocimiento, se busca apoyar su transferencia, divulgación y la producción de alianzas con la comunidad. Esto promueve el acercamiento y el establecimiento de vínculos con actores locales e instituciones, lo cual se asocia a la visión de que el acercamiento del campo académico y el campo de la intervención territorial puede hacer avanzar los estudios, las enseñanzas y las investigaciones científicas y así fortalecer las estrategias privadas y las políticas públicas de desarrollo (precisión de los cuestionamientos, acceso a datos no disponibles para el investigador, intercambios de experiencias disciplinares, involucramiento con la región). Al mismo tiempo, se reconocen demandas territoriales y actores (empresas, municipalidades, ONG, etc.) que buscan acercarse al conocimiento, propiciando los lazos entre universidad y comunidad.

El equipo de investigación trabaja complementando la producción del conocimiento científico y la intervención especializada en proyectos o actividades de extensión. Este contacto con actores locales y regionales, apoyado en trabajos de campo, permite identificar demandas sociales específicas.

\section{La escuela secundaria y las prácticas de sostenibilidad}

El equipo extensionista estuvo integrado por docentes de grado de las áreas de Ingeniería; docentes de posgrado e investigadores del área de Energías Renovables; profesionales de la Arquitectura e Ingeniería; y alumnos de carreras de Ingeniería (Mecánica, Industrial, en Alimentos). A su vez, los integrantes del equipo residen en diversas localidades de la provincia de Buenos Aires, particularmente del noroeste.

La Escuela Agraria fue identificada a partir de contactos previos establecidos con la institución para evaluar el desarrollo de un proyecto de vinculación tecnológica. Fue elegida debido a sus características técnicas, su orientación a la especialidad agraria, su distribución espacial e implantación en la región de influencia de UNNOBA, y se contó con el respaldo y la conformidad de autoridades escolares.

La escuela se ubica en el área periurbana de la localidad de Lincoln, provincia de Buenos Aires, en una región cuyas principales actividades económicas provienen del sector agropecuario. Realiza emprendimientos productivos autosustentables con finalidad pedagógica en zona de chacras, a $6 \mathrm{~km}$ de la ciudad. Desde 2015 la institución funciona en este lugar, donde se nuclean por primera vez las actividades pedagógicas tanto como las productivas. Históricamente, las actividades de aula se realizaban en diversas sedes, en el centro de Lincoln, mientras que en campos o predios rurales variables se efectuaban las actividades de producción. Estas últimas están en proceso de desarrollo y ampliación y son denominadas como "entornos productivos".

Puestos en contacto con directivos y docentes, se comenzó a perfilar un proyecto que ofreciera alternativas y soluciones sostenibles para las necesidades socioambientales y energéticas de la comunidad educativa. Asimismo, se apuntó a difundir en la escuela conceptos de eficiencia energética, nociones de uso de energías renovables y manejo de residuos. Cuestiones necesarias para que los integrantes de la comunidad se apropiaran del proyecto ya que son los encargados de la realización de tareas ordinarias para asegurar el funcionamiento y mantenimiento de las alternativas y soluciones sostenibles. 
La propuesta consistió en jornadas de trabajo conjunto en las cuales los actores aportaron sus experiencias y conocimientos en torno a temáticas predeterminadas, tales como:

- Ambiente y Sostenibilidad.

- Manejo de Residuos y economía circular.

- Eficiencia energética.

- Energías renovables.

Para el desarrollo del proyecto se optó por la metodología de taller. En los procesos de educación popular, el taller es un

"dispositivo de trabajo con grupos, que es limitado en el tiempo y se realiza con determinados objetivos particulares, permitiendo la activación de un proceso pedagógico sustentado en la integración de teoría y práctica, el protagonismo de los participantes, el diálogo de saberes, y la producción colectiva de aprendizajes, operando una transformación en las personas participantes y en la situación de partida". (Cano, 2012:33)

Las actividades de extensión fueron lideradas por los directores del proyecto y contaron con la participación de los docentes-investigadores del equipo. Los alumnos universitarios contribuyeron en las actividades de registro (audiovisual y escrito), observación (con miras a la evaluación interna de cada taller), y moderación (haciendo respetar los tiempos pautados para cada actividad) de los talleres.

Desde su inicio, y en conjunto con los directivos de la escuela, se buscó el diálogo del proyecto con los espacios curriculares con el objetivo de integrar las temáticas preestablecidas con las actividades educativas corrientes y de este modo arribar a las prácticas de sostenibilidad propuestas. En los talleres de extensión se optó por la participación de docentes a cargo de actividades prácticas en los entornos productivos de manera que los profesores contribuyeran y orientaran la articulación de los talleres con los contenidos pedagógicos y las actividades de huerta, animales de granja y agricultura.

El proyecto se estructuró en jornadas de trabajo distribuidas mensualmente y teniendo en cuenta el calendario escolar. La jornada inicial tuvo la finalidad de socializar el propósito del proyecto entre los integrantes de la comunidad educativa y tomar conocimiento de las actividades productivas y pedagógicas planificadas por la escuela para el ciclo lectivo $2019 .^{4}$ Una vez presentadas estas actividades se identificaron los grupos de alumnos y docentes comprometidos con cada una de las tareas (pariciones, limpieza de corrales, invernadero, etc.). A ello se sumó la puesta en conocimiento de las actividades del ciclo lectivo, a fin de no superponer jornadas de extensión con tareas pedagógicas. Se buscó ajustar un cronograma de los encuentros mensuales pautados. Esto permitió delinear estrategias para la elaboración de material didáctico y readecuación de encuentros posteriores.

4) Las actividades de producción consisten en la cría de animales de granja y cultivos de invernadero y de campo, aunque pueden variar de un año a otro en función del rendimiento o las contingencias de temporadas anteriores, o en relación con el presupuesto asignado para ampliar o desarrollar nuevas actividades. Por ejemplo, desde hace varios años la escuela proyecta la instalación de galpones avícolas. Esto todavía no se ha implementado, aunque forma parte de su agenda a largo plazo. 
Las jornadas intermedias consistieron en acciones participativas y talleres en torno a temáticas preestablecidas y/u orientadas a problemáticas emergentes. Los talleres contemplaron una parte introductoria a los temas propuestos a cargo de los integrantes del equipo para luego pasar a la realización de trabajos grupales y/o prácticas con los alumnos, docentes y no docentes de la escuela. En cuanto a la participación del grupo extensionista, los responsables de cada taller variaron y se alternaron en función de las temáticas propuestas y su disponibilidad individual. Esta variabilidad admitió proponer reajustes en el desarrollo de las actividades originalmente propuestas, sin alterar la esencia del proyecto. En cuanto a la participación de los alumnos, las autoridades escolares definieron los grupos participantes conforme a los emergentes y la disponibilidad de docentes a cargo. Se contó con la participación discontinua de diferentes cursos de la escuela secundaria y de alumnos de Tecnicatura de nivel terciario que realizan allí prácticas profesionalizantes. Por un lado, esto aportó diversidad a los talleres y posibilitó que alumnos de distintos cursos y edades fueran partícipes de alguno de los talleres realizados; por otro lado, no se mantuvo un grupo constante (salvo algunas excepciones) a lo largo de todo el proyecto. La participación de los docentes y directivos escolares fue fundamental para el desarrollo del proyecto en general y de las actividades particulares de los talleres y se hizo con la mayor predisposición para el trabajo conjunto.

Los talleres contaron con instancias de auto y coevaluación permanente. Durante el desarrollo del proyecto se implementaron evaluaciones participativas, de las cuales formaron parte los integrantes del equipo y la institución receptora. La evaluación durante la realización del proyecto permitió adecuar o corregir instancias que no habían sido consideradas con anterioridad para reorientarlas en función de los objetivos perseguidos. Además, se incluyó una evaluación final que posibilitó valorar los impactos del proyecto, definir su calidad de funcionamiento y el grado de cumplimiento de los objetivos (Cohen y Franco, 2009; De Santos et al., 2017).

Finalmente, las actividades de difusión procuraron socializar resultados, relevamientos o prácticas, producto de lo trabajado a lo largo del año. Esa socialización se hizo dentro de la comunidad educativa y extensiva a otros actores a través de Radio Universitaria de UNNOBA, páginas web de actividades institucionales de la Universidad, redes sociales de la Escuela Agraria y medios radiales de la localidad de Lincoln.

El proyecto se planteó en diálogo con políticas públicas nacionales, provinciales y municipales. La localización de los ODS, es decir, su aplicación adaptada a cada nivel jurisdiccional dentro de Argentina, ha tenido impulso a distintas escalas. El uso racional y eficiente de la energía, así como el desarrollo de energías renovables han sido políticas de incentivo nacional que tienen eco a nivel provincial, particularmente a través de programas que privilegian acciones sostenibles en edificios de la administración pública y en escuelas. A su vez, los gobiernos locales contribuyen activamente a la difusión y democratización de prácticas de sostenibilidad mediante su vinculación a redes de cooperación entre municipios y acciones locales.

\section{Impactos en la comunidad educativa escolar y al interior del espacio extensionista}

El proyecto apuntó a poner en conocimiento posibles alternativas y soluciones sostenibles para la institución escolar y a concientizar a la comunidad educativa sobre el cuidado del ambiente, el uso de las energías renovables, la eficiencia energética, el manejo de resi- 
duos, etc. Para ello se abordaron diversos ejes orientados a atender diversas necesidades:

- Pedagógicas: a través de la difusión de tecnologías, conocimientos y hábitos de sostenibilidad ambiental y energética en una comunidad educativa que, por su orientación agraria, realiza actividades de producción primaria.

- Socioambientales: mediante propuestas de reutilización de residuos orgánicos provenientes del comedor escolar. La escuela cuenta con una población de 120 alumnos (de los cuales 15 son internos pupilos) y un plantel de 70 personas entre docentes y no docentes. Un comedor comunitario provee 4 comidas diarias a los alumnos internados y 3 comidas diarias al resto de alumnos y docentes.

- Productivas: apuesta por la producción agroindustrial sustentable. En la escuela se realizan emprendimientos productivos de cría de animales y cultivos de invernadero en un predio de 6 hectáreas. A ello se suman 7 hectáreas para cultivos de rotación (trigo-soja-maíz) y 6 hectáreas de pasturas.

- Energéticas: propuestas de producción de energía térmica que reemplace y/o complemente el consumo eléctrico y de gas (Gas Licuado de Petróleo [GLP]) envasado y a granel. La electrificación del predio escolar proviene de la red eléctrica; el agua se extrae de la napa subterránea con un sistema de bomba eléctrica; y dado que no hay red de gas en áreas suburbanas, se utiliza GLP. El gas destinado a la alimentación de calderas para calefacción y para cocción de alimentos, es GLP a granel (zeppelin) proporcionado por la Dirección de Cultura y Educación de la Provincia de Buenos Aires. Adicionalmente, se requiere GLP envasado (tubos), que es solventado con recursos propios de la escuela, para calefaccionar madrigueras de cerdos y galpones avícolas durante el período invernal.

La producción de bioenergía en ámbitos rurales es una práctica que se va extendiendo paulatinamente. Desde 2016 el Ministerio de Agricultura de la Nación destinó fondos para la instalación de biodigestores ${ }^{5}$ en 12 escuelas rurales del país. Solo dos escuelas bonaerenses (de las localidades de Las Flores y Navarro) fueron beneficiadas. En el noroeste de la provincia de Buenos Aires se han relevado otros $\operatorname{casos}^{6}$ de instituciones escolares que llevaron adelante proyectos de esta naturaleza, en su mayoría con la finalidad de producir biogás para sistemas de cocción, y se detectaron dificultades operativas (no tanto tecnológicas) para sostener en el tiempo las actividades que conlleva el mantenimiento en funciones de un biodigestor. Por esta razón, en el proyecto se hizo hincapié en la participación de la comunidad educativa para la concientización del cuidado del ambiente y el uso de energías renovables.

Dentro del grupo extensionista esta experiencia significó la generación de vínculos con un actor fundamental para el desarrollo de actividades ambientales: una escuela secundaria de

5) Los biodigestores son contenedores donde se deposita materia orgánica que segregue bacterias (desechos vegetales, excremento) dentro de una disolución con agua. Esta mezcla, mediante la fermentación anaeróbica de los microorganismos, se degrada obteniendo como producto gas metano (biogás) y abono orgánico. Los biodigestores representan una alternativa para el tratamiento de los desechos orgánicos de las explotaciones agropecuarias ya que permiten disminuir la carga contaminante, mejorar la capacidad fertilizante, y generar un gas combustible que puede reemplazar al gas natural (Universidad Nacional de Cuyo, s.f.).

6) Escuela de la Familia Agrícola de Acevedo (Pergamino) y Escuela Agrotécnica de Duggan (San Antonio de Areco). Durante 2018, la Dirección de Sustentabilidad, Medio Ambiente y cambio Climático del Ministerio de Agroindustria de la Provincia de Buenos Aires llevó adelante un Programa de Biodigestores en Escuelas Rurales y construyó biodigestores en escuelas de La Plata, Bavio y L. N. Alem. 
gestión estatal que forma técnicos ${ }^{7}$ y donde también realizan prácticas profesionales alumnos de nivel terciario de una Tecnicatura en Producción Agrícolo-ganadera. El desarrollo de los talleres a lo largo del año escolar facilitó el establecimiento de lazos fructíferos en un ámbito franco con el plantel docente, directivo y la población de alumnos.

Para el equipo universitario, el proyecto representó un aprendizaje con diversas aristas. Por un lado, se tuvo contacto con la realidad socioeducativa del contexto público escolar en una institución de doble jornada, con comedor comunitario e internado para alumnos no residentes en la localidad. Esto supuso un gran contraste con la población y la modalidad de la formación universitaria. Por otro lado, se recolectaron saberes y experiencias aportadas por alumnos y docentes sobre el manejo y funcionamiento del edificio escolar y los entornos productivos. Este conocimiento permitió idear propuestas de sostenibilidad a partir de los recursos existentes (venta de plantines en portamacetas biodegradables; construcción de un biodigestor pedagógico alimentado por estiércol de los animales de granja y desechos orgánicos del comedor; instalación de panel solar para alimentación de un boyero y para iluminación de un galpón, etc.). La experiencia también tuvo impactos en los procesos de investigación, ya que la formulación de los talleres significó una transferencia de conocimientos específicos que debieron ser adaptados al contexto escolar (a través de juegos grupales, cuestionarios, videos, etc.) y a las particularidades de la institución receptora.

También se presentaron algunos obstáculos y cuestiones a reformular. Como se mencionó, la participación de los alumnos fue variable y heterogénea, definida por las autoridades escolares en función de los emergentes y la disponibilidad de docentes a cargo. Aun la presencia de docentes en los talleres fue diversa y dependió de los cronogramas y horarios de clase. A la vez, hubo cambio de autoridades directivas en el lapso transcurrido entre los primeros contactos establecidos con la escuela y el inicio del proyecto, pero sin que esto representara modificación alguna a la finalidad del proyecto más que las presentaciones correspondientes y la reafirmación de acuerdos previos. Para el desarrollo de los talleres se confeccionaron guiones que organizaron y pautaron las actividades y los tiempos asignados para cada una. Esas guías fueron cuidadosamente delineadas, teniendo en cuenta los conocimientos a transferir a través de una introducción teórica, las actividades propuestas -relacionadas con el tema prefijado-, la puesta en común de experiencias, saberes y trayectorias, y un cierre que rescató la idea de construcción colectiva. A raíz de los escenarios cambiantes que se presentaron en cada uno de los talleres se debió ajustar el guion en cuanto a los tiempos, orden de las actividades y roles dentro del equipo universitario. Pero al desarrollo interno de los talleres se agregaron gestiones imprevistas para montar el espacio de trabajo (con proyector, sillas, mesas, pizarra y materiales varios), la confirmación de los cursos y docentes asistentes, y el control de los materiales y equipamientos suministrados por la escuela. Es decir que se trabajó en un escenario de cambios y desafíos constantes, lo que obligó a generar propuestas de adaptación para afrontar las contingencias, desde el ajuste de contenidos y actividades según el grupo participante, hasta la utilización de espa-

7) La Educación Secundaria Agraria es una de las alternativas de la Modalidad Educación Técnico Profesional en el marco de la Educación Secundaria obligatoria en la provincia de Buenos Aires. En la culminación del $6^{\circ}$ año, los alumnos reciben el título de Bachiller Agrario, y al finalizar el $7^{\circ}$ año, los egresados obtienen el título de Técnico en Producción Agraria con la especificación de la orientación que corresponda (Dirección General de Cultura y Educación, s.f.). 
cios alternativos y/o variables. Esto afianzó el vínculo al interior del equipo extensionista y subrayó su cualidad de adaptación a los emergentes, cuestión que requirió tanto de la capacidad de trabajo — individual y colectiva — como de las sinergias con la comunidad educativa.

\section{Reflexiones finales}

En el siglo XXI Argentina enfrenta los desafíos de disminuir la utilización de energías fósiles y privilegiar la sostenibilidad ambiental y energética. Progresivamente se observa un aprendizaje por parte de los actores para conformar mecanismos de regulación a favor de un desarrollo sostenible. Esto se ve reflejado en nuevas experiencias, tanto a escala nacional, desde iniciativas públicas a partir de programas y proyectos de energía renovable, ${ }^{8}$ como a nivel provincial y local, donde el nuevo rol de actores locales cobra especial protagonismo.

La UNNOBA transita una expansión de la actividad de extensión que incluye convocatorias anuales para el desarrollo de proyectos, capacitaciones, mayores presupuestos, así como el incremento en la presentación de nuevas propuestas anuales. Todo ello con el objetivo de realizar acciones que generen impactos en la calidad de vida de las comunidades de la región. En particular la cuestión ambiental es abordada por la institución desde áreas de gestión específicas, programas de apoyo a la vinculación comunitaria, contenidos académicos y líneas de investigación. Desde este ámbito —la investigación- se conformó un equipo extensionista caracterizado por la multidisciplinariedad y la experiencia previa en trabajos de campo y formación de vínculos con actores locales y regionales.

El proyecto aquí presentado consistió en trasladar a una comunidad educativa de orientación agraria temas y problemáticas estudiados e investigados por el equipo donde la sostenibilidad ambiental se tradujera en prácticas cotidianas, fácilmente abordables por la comunidad en general y por la escolar en especial por tratarse de una institución que realiza actividades pedagógicas ligadas a la cuestión ambiental.

La ejecución y seguimiento de este proyecto permitió:

- Elaborar propuestas y construir espacios de intercambio a partir de necesidades o requerimientos manifestados por alumnos, docentes y directivos.

- Que las prácticas adquiridas puedan replicarse y deriven en nuevas posibilidades laborales y productivas para los actores involucrados.

- Que esta experiencia transfiera un conjunto de herramientas con la finalidad de implementar prácticas sostenibles y eficientes que aseguren la optimización de los recursos disponibles en la escuela.

- Considerar cambios y/o mejoras y nuevas instancias de implementación para extender la propuesta a comunidades educativas de otros contextos (urbanos, con poblaciones de riesgo, etcétera).

- Recopilar experiencias que permitan acrecentar el conocimiento y generar un modelo para futuras iniciativas.

8) Proyecto de Energías Renovables en Mercados Rurales (PERMER I y II), Generación de Energía Eléctrica a partir de Fuentes Renovables (GENREN I y II), Programa Nacional de Uso Racional y Eficiente de la Energía (PRONUREE), Proyecto para la Promoción de la Energía Derivada de Biomasa (PROBIOMASA). 
La irrupción de la pandemia por COVID-19 ha puesto en suspenso la continuidad de estas actividades y su aplicación extensiva a otros ámbitos. No obstante, consideramos que la incorporación de prácticas de sostenibilidad en ámbitos educativos contribuiría con objetivos de lucha contra el cambio climático, aumentaría la seguridad energética y favorecería la inclusión social de sectores vulnerables.

\section{Referencias bibliográficas}

Cano, A. (2012). La metodología de taller en los procesos de educación popular. Revista Latinoamericana de Metodología de las Ciencias Sociales, 2(2), 22-51. Memoria Académica. http://www.memoria.fahce.unlp.edu. ar/art_revistas/pr.5653/pr.5653.pdf

Carrasco, J. C.; Cassina, R. y Tommasino, H. (2009). Extensión en obra. Experiencias, reflexiones, metodologías y abordajes en extensión universitaria. Universidad de la República. http://beu.extension.unicen.edu.ar/xmlui/ bitstream/handle/123456789/201/Extensión\%20en\%20obra\%20Experiencias\%2c\%20reflexiones\%2c\%20 metodologías\%20y\%20\%20abordajes\%20en\%20extensión\%20universitaria.pdf?sequence=1 \&isAllowed=y Carrizo, S. y Gil, S. (2018). Servicios sostenibles frente a la pobreza energética en Argentina. Energía Estratégica. http://www.energiaestrategica.com/opinion-como-enfrentar-a-la-pobreza-energetica-argentina-conenergias-renovables/

Cohen, E. y Franco, R. (2009). Evaluación de proyectos sociales. Siglo XXI Editores.

De Santos, C.; Stevenazzi, F., Romero, F. S.; Moratti Serrichio, F.; Tommasino, H.; Almada, J.; Tomatis, K.; Bermúdez, L.; Carignano, M.; Bonicatto, M.; Gómez, M. I.; González, M. N.; Grabino, V. y Calvo, V. (2017). Fronteras universitarias en el Mercosur: debates sobre la evaluación en prácticas en extensión. Universidad Nacional de Córdoba. http://beu.extension.unicen.edu.ar/xmlui/bitstream/handle/123456789/190/Fronteras\%20universitarias\%20en\%20el\%20Mercosur\%20debates\%20sobre\%20la\%20evaluación\%20en\%20 prácticas\%20en\%20extensión.\%20\%281\%29.pdf?sequence=1\&isAllowed=y

Dirección de Sustentabilidad, Medio Ambiente y Cambio Climático, Provincia de Buenos Aires (2018). Instructivo Biodigestor. Conceptos básicos y pasos para la construcción y puesta en funcionamiento de un biodigestor para aplicación en escuelas rurales y agrotécnicas, o bien para autoconsumo en instalaciones domiciliarias. https://www.gba.gob.ar/sites/default/files/agroindustria/docs/Instructivo_Biodigestor.pdf

Dirección General de Cultura y Educación (s.f.) http://abc.gob.ar/secretarias/niveles/educacion_secundaria Huerga, I.; Butti, M. y Venturelli, L. (2014). Biodigestores de pequeña escala. Un análisis práctico sobre su factibilidad. INTA Oliveros. https://es.scribd.com/document/199852962/Biodigestor-DE-PEQUENA-ESCALA-HUERGA-BUTTI-VENTURELLI

Kozak, D. y Romanello, L. (2012). Sustentabilidad en arquitectura 2. Consejo Profesional de Arquitectura y Urbanismo, Buenos Aires.

Medina, J. M. (2018). Extensión crítica: Construcción de una universidad en contexto: sistematización de experiencias de gestión y territorio de la Universidad Nacional de Rosario. UNR Editora.

ONU - Organización de las Naciones Unidas (1987). Bruntland Report, Our Common future. http://www.undocuments.net/our-common-future.pdf

ONU - Organización de las Naciones Unidas (s.f.). https://www.un.org/sustainabledevelopment/es/objetivosde-desarrollo-sostenible

Organización de las Naciones Unidas para la Educación, la Ciencia y la Cultura (1998). Conferencia Mundial sobre la Educación Superior. https://unesdoc.unesco.org/ark:/48223/pf0000116720 
Thema, M.; Sterner, M.; Lenck, T. y Götz, P. (2016). Necessity and impact of power-to-gas on energy transition in Germany Energy. Procedia, 99, 392-400. https://www.sciencedirect.com/science/article/pii/ S1876610216310906?via\%3Dihub

Universidad Nacional de Cuyo (s.f.). Instituto de Energía. Programa de Biogás. http://www.imd.uncu.edu.ar/ upload/manual-uso-biodigestor.pdf

UNNOBA - Universidad Nacional del Noroeste de la Provincia de Buenos Aires (2009). Estatuto. Título V: De la función social de la Universidad. Capítulo II, De la Extensión Universitaria (p. 10).

UNNOBA - Universidad Nacional del Noroeste de la Provincia de Buenos Aires (s.f.). Extensión Universitaria https://www.unnoba.edu.ar/extension/informacion-general/ 